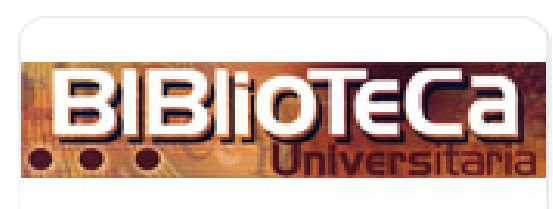

Biblioteca Universitaria

ISSN: 0187-750X

public@dgb.unam.mx

Universidad Nacional Autónoma de México

México

Guzmán Sánchez, María Victoria; Trujillo Cancino, José Luis Los mapas bibliométricos o mapas de la ciencia: una herramienta útil para desarrollar estudios métricos de información

Biblioteca Universitaria, vol. 16, núm. 2, julio-diciembre, 2013, pp. 95-108

Universidad Nacional Autónoma de México

Distrito Federal, México

Disponible en: http://www.redalyc.org/articulo.oa?id=28529572002

- Cómo citar el artículo

- Número completo

- Más información del artículo

- Página de la revista en redalyc.org

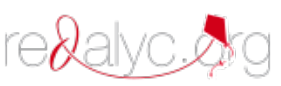

Sistema de Información Científica

Red de Revistas Científicas de América Latina, el Caribe, España y Portugal

Proyecto académico sin fines de lucro, desarrollado bajo la iniciativa de acceso abierto 


\title{
Los mapas bibliométricos o mapas de la ciencia: una herramienta útill para desarrollar estudios métricos de información
}

\author{
Bibliometric maps or maps of science: a useful tool for developing \\ metric studies of information
}

María Victoria Guzmán Sánchez", José Luis Trujillo Cancino**

\section{Resumen}

En el presente trabajo se hace una reflexión en torno a los mapas bibliométricos o mapas de la ciencia, de los cuales podemos decir que son diagramas que representan las palabras, ideas, tareas, u otros conceptos ligados y dispuestos radialmente alrededor de una palabra clave o de una idea central. Se utilizan para la generación, visualización, estructura y clasificación taxonómica de las ideas, así como ayuda interna para el estudio, organización, solución de problemas y toma de decisiones en una organización.

Palabras clave: Mapas bibliométricos, mapas de la ciencia, bibliometría.

\section{Abstract}

In this paper a reflection is made on bibliometric maps or maps of science, which are diagrams representing the words, ideas, tasks or other items linked to and arranged radially around a keyword or a central idea. They are used for the generation, visualization, structure and taxonomic classification of ideas as well as an internal support for the study, organization, problem solving and decision making in an organization.

KEYWORDS: Bibliometric maps, maps of science, bibliometrics.

* Instituto Finlay, Centro de Investigación-Producción de Vacunas y Sueros. Av. 27, no. 19805, La Lisa, A.P. 16017, Cod. 11600, Ciudad de la Habana, Cuba. Correo electrónico: mvguzman@finlay.edu.cu.

** Departamento de Suscripciones, Subdirección de Servicios de Información Especializada, Dirección General de Bibliotecas de la Universidad Nacional Autónoma de México (UNAM). Circuito de la Investigación Científica S/N, Ciudad Universitaria, 04510, México, D.F. Correo electrónico: joseltca@unam.mx. 


\section{Introducción}

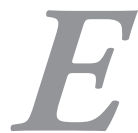

I ser humano desde tiempos muy remotos ha tenido la necesidad de cartografiar su pensamiento y plasmar hacia el exterior esa representación, esta fue la forma en la que nacieron los primeros mapas en las civilizaciones más antiguas; estos mapas fueron utilizados en primer instancia para guiar a los ejércitos en las campañas de conquista y posteriormente sirvieron para los comerciantes.

\section{Definición}

Según el Diccionario de la Real Academia Española, los mapas son:

1. m. Representación geográfica de la Tierra o parte de ella en una superficie plana.

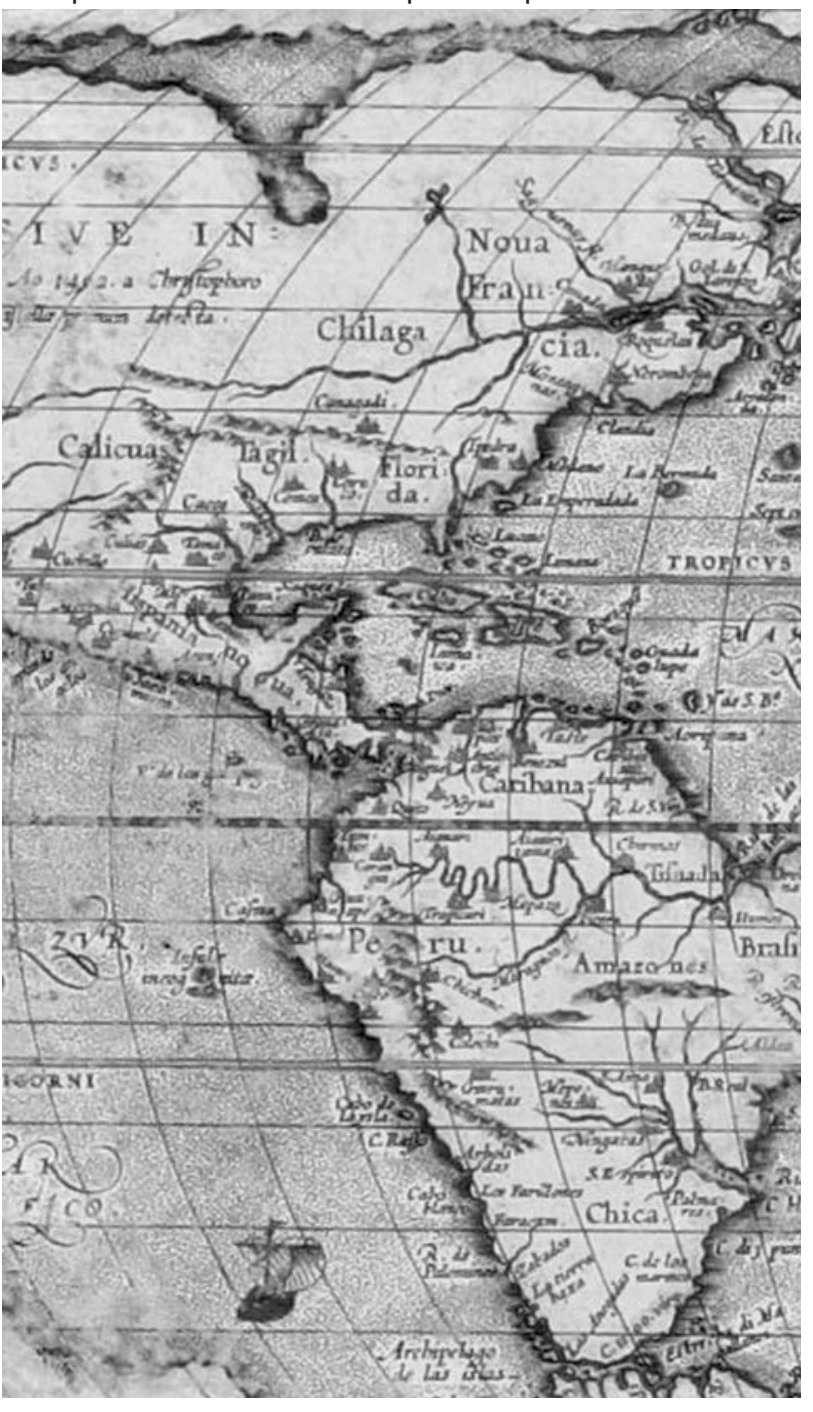

2. m. Representación geográfica de una parte de la superficie terrestre, en la que se da información relativa a una ciencia determinada. Mapa lingüístico, topográfico, demográfico. ${ }^{1}$

Pero para Jazmín Sambrano el mapa es una "representación simbólica" de la realidad exterior, es la manera de cómo un ser humano interioriza primero y exterioriza después su concepto del mundo. Los individuos necesitamos orientarnos, ubicar y registrar los acontecimientos de los hechos, las cosas, los animales, las interacciones, el tiempo, el espacio, la realidad en la que se vive.

Por lo tanto, el mapa es una manera de generar, registrar y asociar ideas, tal como lo procesa el cerebro humano, para plasmarlas en papel. Puede considerarse como una forma de organización, aplicación, explicación, asimilación y modificación de conocimientos para los fines planteados. ${ }^{2}$

\section{Los mapas del conocimiento}

Por otra parte, hay que situar los mapas del conocimiento en los trabajos de Joseph D. Novak en los primeros años de la década de los 60's del siglo pasado. El concepto de mapeo refiere a la técnica de visualización con una larga tradición en el contexto educativo. Esta es una actividad derivada de la investigación en psicología que sirve para representar el conocimiento, ideas, convicciones y creencias; esta técnica fue inventada por Novak y Gowin, basada en la idea de Ausubel. ${ }^{3}$ El concepto de mapa acorde a estos autores lo entendemos como un arreglo espacial que representa los elementos de los conocimientos por medio de nodos y enlaces direccionalmente etiquetados o con nombre; en este caso los nodos representan ideas, conceptos, creencias y las relaciones de vínculos entre ellos. ${ }^{4}$

Real Academia Española. Diccionario de la lengua española [en línea]. 22 ed. <http://lema.rae.es/drae/>

2 Sambrano, Jazmin, Steiner, Alicia. Mapas mentales: agenda para el éxito.

3 Chen, Nian-Shing, Kinshuk, Wei, Chung-Wang, Chen, Hong-Jhe. Mining e-Learning domain concept map from academic articles, p. 1010.

Tergan, Sigmar-Olaf. Digital Concept Maps for Managing 
Estos mapas son diagramas que representan las palabras, ideas, tareas $u$ otros conceptos ligados y dispuestos radialmente alrededor de una palabra clave o de una idea central. Se utilizan para la generación, visualización, estructura y clasificación taxonómica de las ideas, así como ayuda interna para el estudio, organización, solución de problemas y toma de decisiones en una organización. ${ }^{5}$

Para Small un mapa es una representación espacial de los campos, de las disciplinas, especialidades y de los trabajos de los autores que están relacionados entre sí, como se muestra por su proximidad física y ubicaciones relativas, de forma análoga a los mapas geográficos, los cuales muestran las relaciones de las características políticas o físicas en la Tierra. ${ }^{6}$ Por su parte, Janecek plantea que las interfaces visuales son herramientas potencialmente útiles para que los usuarios puedan explorar una representación y descubrir información para ellos relevantes. ${ }^{7}$

La visualización de información no es el resultado inmediato del acto de "ver". Requiere transformar datos abstractos y fenómenos complejos de la realidad en mensajes visibles. Esto hace posible que los individuos vean con sus propios ojos datos y fenómenos que yacen ocultos y que no son directamente aprehensibles. En otras palabras, la visualización consiste en hacer visible para nuestra mente aquello que no es visible para nuestros ojos. ${ }^{8}$

\section{Potencialidades de los mapas bibliométricos}

Los mapas bibliométricos son una poderosa herramienta para el estudio de la estructura y dinamismo de un campo científico. Los investigadores pueden utilizar los mapas bibliométricos para obtener un mejor entendimiento en el campo en el cual están trabajando. En

Knowledge and Information, p. 187.

5 Solutecia [en línea]. Soluciones Tecnológicas en Inteligencia

Artificial. <http://portal.solutecia.com/producto-sofia/129mapa-de-conocimiento>

6 Small, Henry. Visualizing science by citation mapping, p. 799.

7 JANECEK, Paul, Pu, Pearl. An evaluation of semantic fisheye views for opportunistic search in an annotated image collection, p. 42.

8 Miguel, Sandra Edith, CAPrile, Lorena, Jorouera-VIDAL, Israel. Análisis de co-términos y de redes sociales para la generación de mapas temáticos, p. 639. adición, estos mapas pueden proveer de un valor adicional para propósitos de política científica.

Se distinguen varios tipos de mapas bibliométricos, cada uno visualiza la estructura de un campo científico desde diferentes puntos de vista; algunos mapas por ejemplo muestran la relación entre autores o revistas basados en la co-citacion; otros mapas muestran la relación entre palabras o palabras clave basadas en la co-ocurrencia.

Los mapas posteriores son usualmente referidos como mapas de co-palabra o también denominados mapas de términos; entendiéndose a éstos como una palabra o frase que refiere el concepto a un dominio específico. ${ }^{9}$

\section{Uso de los mapas bibliométricos o mapas de la ciencia}

Los mapas de la ciencia son la representación gráfica en dos o tres dimensiones de un campo de la ciencia. Ofrecen un paisaje o panorama de ésta, donde los elementos del mapa se refieren a temas o términos en el campo asignado, como las ciudades en un mapa geográfico. En estos mapas los elementos se colocan relacionándose unos con otros, de tal manera que aquellos temas cognitivos que están relacionados entre sí, están situados en las proximidades, y los que no están relacionados o apenas lo están se encuentran distantes.

Los mapas de la ciencia más conocidos son aquellos basados en los datos bibliográficos, a los cuales se denominan mapas bibliométricos de la ciencia; como supone la literatura cientifica, sirven para representar la actividad de una ciencia. Un mapa basado en los datos de la publicación científica dentro de un campo de la ciencia puede considerarse como un elemento para representar la estructura de la disciplina o ciencia. Dependerá de la información (elementos del registro bibliográfico) utilizada para construir el mapa para determinar qué tipo de estructura será generada y lo bien construida que quede, es decir, hasta qué punto la estructura es reconocida por el experto de campo.

\footnotetext{
9 Van Eck, Nees Jan, Waltman, Ludo, Noyons, Ed C. M., BuTER, Reindert K. Automatic term identification for bibliometric mapping, p. 582.
} 
La mayoría de los mapas de la ciencia están construidos por el principio de información de co-ocurrencias, es decir, la ocurrencia o aparición de más de dos términos que se presentan en un documento, mucho antes de que se identificaran por estar estrechamente relacionados. El principio de asignación de ciencia dicta que entre más cerca se localicen dos elementos relacionados más cerca entre sí se sitúan en un mapa.

El análisis de co-términos es una variante del análisis de co-palabras (un término puede estar compuesto de varias palabras). En cualquier caso, se trata de una técnica de análisis de contenido que usa patrones de co-ocurrencia de pares de términos o palabras dentro de un corpus textual, para identificar las relaciones entre las ideas en un dominio determinado. ${ }^{10}$

Existen muchos datos en un registro bibliográfico que pueden utilizarse para generar una estructura. Cada elemento revela una estructura específica, única en un sentido, pero siempre en relación con las estructuras basadas en otros elementos.

Un registro bibliográfico contiene un intervalo de elementos; los más importantes son:

- Autor(es) de una publicación.

- Título de la publicación.

- Fuente en la que se publica el documento, por ejemplo, una revista científica, un proceeding, un libro.

- Año de publicación.

- Dirección (es) de los primeros autores.

- Resumen de la publicación.

En bases de datos bibliográficas especializadas puede incluirse otra información como:

- Referencias citadas.

- Información del editor o de la fuente.

- Palabras clave proporcionadas por el autor, la revista o el editor.

- Códigos de clasificación agregados por el creador de la base de datos.

10 Miguel, Sandra Edith, Caprile, Lorena, Jorquera-Vidal, Israel, op. cit., p. 640.
- Indexado de términos agregados por el creador de la base de datos. ${ }^{11}$

El mapeo puede considerarse como un puente para relacionar el campo de investigación como la visualización del conocimiento y la recuperación de información. Los mapas bibliométricos son resúmenes gráficos de documentos obtenidos de una base de datos de citas, palabras o frases, o algunos elementos bibliométricos. Parte de su atractivo se basa en las características compartidas con sus contrapartes geográficas, que les permite ser referido como "paisajes de la ciencia". ${ }^{2}$

\section{Características y uso de los EMI en el mapeo científico}

Los estudios métricos de la información (EMI) son una herramienta eficaz para detectar y analizar el surgimiento de nuevas tecnologías. Éstos estudios consisten en el análisis estadístico de las publicaciones científicas, que adopta los indicadores cuantitativos de desempeño, lo cual ayuda a superar el inconveniente de la subjetividad en la evaluación por pares y las opiniones de expertos; esta herramienta se ha utilizado para evaluar el rendimiento de la investigación en una cantidad cada vez mayor y una gran variedad de estudios; además, han sentado las bases para la aplicación de los análisis básicos que evalúan las nuevas áreas de investigación. ${ }^{13}$

Para César Macías Chapula los EMI son una herramienta mediante la cual se pueden observar el estado de la ciencia y la tecnología a través de la producción global de la literatura científica en un nivel dado de especialización; los EMI son igualmente apropiados para realizar macro análisis, por ejemplo, de la participación de un país (o región) determinado en la producción científica durante un periodo específico, y para los micro análisis, por ejemplo, del

11 Noyons, Ed C. M. Bibliometric mapping of science in a science policy context, p. 84

12 Buter, Reindert K., Noyons, Ed C. M., Van Mackelenbergh, M., Laine, T. Combining concept maps and bibliometric maps: First explorations, p. 377.

13 CHEN, Kaihua, GUAN, Jiancheng. A bibliometric investigation of research performance in emerging nanobiopharmaceuticals, p. 234 
papel de un autvor (o institución) concreto en la elaboración de artículos en un campo específico de la ciencia. En combinación con otros indicadores los EMI pueden ayudar a valorar el estado actual de la ciencia y apoyar en la toma de decisiones y en la dirección de la investigación. ${ }^{14}$

Es importante destacar la evaluación de programas de investigación y desarrollo (ID) que ha sobresalido desde la década de los $80^{\prime}$ del siglo pasado. Al introducirse el concepto de gerencia o administración estratégica de la investigación, ya que los programas de ID pueden facilitar proyectos individuales, la evaluación es un aspecto importante de la gerencia en un sistema de investigación e innovación. ${ }^{15}$

Los estudios cuantitativos de la ciencia han desarrollado métodos y herramientas para comprender mejor la organización de los campos de la ciencia y su evolución. Los mapas globales de la ciencia se han convertido recientemente en herramientas viables que ofrecen una vista preliminar general de los campos científicos. ${ }^{16}$

En general, un mapa de la ciencia se compone de un conjunto de elementos; estos elementos pueden ser campos o disciplinas científicas, revistas, documentos o cualquier otra unidad que representa una partición de la ciencia. Las características que diferencian a un mapa de un sistema de clasificación simple son:

a. la visualización de los elementos, comúnmente representados mediante la localización de cada uno de los elementos en el espacio bidimensional, y

b. la vinculación explícita de elementos pares (o afines) en virtud de la relaciones entre ellos. ${ }^{17}$

14 Macías-Chapula, César A. Papel de la informetría y de la cienciometría y su perspectiva nacional e internacional, p. 37.

15 Yoon, Byungun, Lee, SungJoo, Lee, GwangheE. Development and application of a keyword-based knowledge map for effective R\&D planning, p. 804.

16 Grauwin, Sebastián, Jensen, Pablo. Mapping scientific institutions, p. 943.

17 KLavans, Richard, Boyack, Kevin W. Toward a consensus map of science, p. 456.
Entre los principales objetos de estudio de los EMI planteados en los mapas de científicos encontramos:

- Los aspectos estadísticos del lenguaje y la frecuencia de uso de las palabras y frases, tanto en textos redactados en lenguaje natural como en otros medios impresos y electrónicos.

- Las características de la productividad autoral, medida por el número de documentos publicados o por el grado de colaboración.

- Las características de las fuentes publicadas, incluyendo la distribución de los documentos por disciplinas.

- Los análisis de citas, teniendo en cuenta la distribución por autores, por tipo de documento, por instituciones y por países.

- La obsolescencia de la literatura, en virtud de la medición de su uso y de la frecuencia con que se cita.

- El incremento de la literatura por temas. ${ }^{18}$

\section{Herramientas usadas para el desarrollo de mapas de la ciencia}

Existen varias herramientas para el desarrollo de mapas bibliométricos o de la ciencia; entre los más conocidos encontramos softwares como Pajek, vosviewer y Viscovery somine ${ }^{\circledR}$, que analizaremos con mayor profundidad en el presente artículo.

Pajek es un programa para Windows, que sirve para analizar y visualizar grandes redes a partir de colaboraciones simbolizables en una malla similar a una tela de araña, con relaciones horizontales. Este software es de acceso libre y fue desarrollado por Vladimir Batagelj, Andrej Mrvar y con la contribución de Matjaz Zaverznik en la Universidad de Ljubljana, en Eslovenia (http://vlado.fmf.uni-lj.si/pub/networks/pajek/). Los principales objetivos del diseño con Pajek son:

- Desarrollar grandes redes a partir de redes pequeñas; asimismo, visualizar gráficamente herramientas y técnicas científicas basadas en la Teoría de Grafos.

18 TAgue-Sutcliffe, Jean. Introducción a la informetría, p. 27. 
- Identificar los cálculos de densidad, intermediación y proximidad científica de las cooperaciones. ${ }^{19}$

- Proporcionar al usuario una herramienta de visualización de gran alcance.

- Aplicar una selección eficiente de algoritmos de redes.

En Pajek se pueden manejar múltiples redes simultáneamente y redes con eventos en el tiempo. Los eventos en el tiempo resumen el desarrollo o evolución de las redes a través del tiempo (usando unas diferencias de horario como indicadores). En Pajek se pueden analizar redes con más de un millón de nodos; la disposición de memoria en el equipo establece el límite real. Para ahorrar memoria los nombres y etiquetas de los nodos no se mantienen en las redes muy grandes, pero se pueden adjuntar más adelante a las pequeñas subredes. ${ }^{20}$

vosviewer (http://www.vosviewer.com/) es un software desarrollado en el Centro de Estudios Científicos y Tecnológicos (CWTS) de la Universidad de Leiden (Holanda); éste es un programa de ordenador disponible libremente, está diseñado principalmente para ser utilizado en el análisis de redes bibliométricas.

VOSViewer puede servir para crear mapas de publicaciones, autores o revistas sobre la base de una red de co-citación, o para crear mapas de palabras clave basadas en una red de co-ocurrencia; además, ofrece un visualizador que permite examinarlos con todo detalle, ofreciendo la posibilidad de exhibirlos de diferentes modos, cada uno enfatizando algún aspecto. Adicionalmente ofrece la funcionalidad de enfoque, bajar/subir y de búsqueda, por lo que facilita la vista detallada; en definitiva, sirve para mapas conteniendo un número moderadamente grande de temas (al menos 100). La

19 Luiz Pinto, Adilson, Moreiro González, José Antonio, OliVeira de Mera Gusmão, Alexandre. Análisis de redes sociales a partir de recursos web y de bases de datos especializadas en literatura científica, p. 144

20 Alonso Moreno, Álvaro, González Hernández, Octavio Miguel, Lavera Ulloa, Iván. Proyecto Krowface: interfaz de simulación de redes sociales [en línea]. Sistemas Informáticos, Curso 2011-2012. <http://eprints.ucm.es/16105/1/MEMORIA_FINAL_ENTREGA.pd> mayoría de los programas hasta ahora disponibles no exhiben los mapas de modo satisfactorio. ${ }^{21}$

Por otra parte, vosviewer se puede utilizar para crear mapas basados en datos de la WWW. Los mapas se crean utilizando la técnica de mapeo y de agrupación VOS. Una vez creados se pueden mostrar en varias formas diferentes; en aspectos tales como vista de la etiqueta, vista de densidad, vista de la densidad del cúmulo y vista de dispersión, cada uno enfatizando un aspecto diferente del mapa; además, este software ofrece funcionalidades como zoom, desplazamiento y búsqueda, lo que facilita el realizar exámenes detallados en un mapa. ${ }^{22}$

El Viscovery SOMine ${ }^{\circledR}$ es una aplicación de escritorio para la exploración de minería de datos, análisis de agrupamiento visual, creación de perfiles estadísticos, segmentación y clasificación basada en los mapas auto-organizados (SOM, por sus siglas en inglés), y de estadística clásica en un ambiente de trabajo intuitivo. Desde su primera versión en 1996, Viscovery sомine ${ }^{\circledR}$ (http://www.viscovery.net/somine/) se ha convertido en un estándar comercial para la minería de datos sobre la base SOM; este producto es usado en un gran número de empresas, consultorías y laboratorios, y muchas universidades en todo el mundo hacen uso de SOMine ${ }^{\circledR}$ para sus tareas analíticas. ${ }^{23}$

El Viscovery Somine ${ }^{\circledR}$ organiza " $n$ " dimensión de datos en mapas, con base en su igualdad o similitud; el mapa resultante puede ser usado para identificar y evaluar características ocultas en los datos. La principal arma de Viscovery somine es el despliegue visual de los mapas generados por la red SOM; el sistema permite interactuar con el mapa de tal manera que es posible obtener los datos que contienen las neuronas o nodos, además permite el despliegue de los distintos algoritmos de visualización

21 Van Eck, Nees Jan, WaLtMAN, Ludo. VOSviewer Manual [en línea]. Universiteit Leiden: Erasmus Universiteit Rotterdam, 1 January 2013, p. 3. <http://www.vosviewer.com/documentation/Manual_VOSviewer_1.5.4.pdf>

22 VOSWiewer [en línea]. Universiteit Leiden, 2013. <http:// www.vosviewer.com/>

23 Viscovery SOMine 5.2 Explorative data mining based on SOMs and statistics [en línea] Viena, 2012. <http://www.viscovery.net/welcome> 
y de los clusters o conglomerados, entre otras funciones; pero ¿̇qué son los mapas auto-organizados?

Teuvo Kohonen, profesor de la Facultad de Ciencias de la Información (Helsinki University of Technology), presentó entre 1981/82 un modelo de red neuronal artificial con capacidad de generar de manera automática agrupaciones de datos multidimensionales y proyectar dichas agrupaciones en mapas bidimensionales de manera que las relaciones de similitud entre los datos se representan por la cercanía de sus proyecciones en los mapas. ${ }^{24}$

Esto quiere decir que de manera automática se clasifican los conjuntos de datos, de los que no se conoce a priori ningún tipo de organización; esta red debe descubrir rasgos comunes, regularidades, correlaciones o categorías en los datos de entrada, e incorporarlos a su estructura interna de conexiones. Se dice, por tanto, que las neuronas deben auto-organizarse en función de los estímulos (datos) procedentes del exterior. ${ }^{25}$

El algoritmo de aprendizaje de la red SOM está basado en el aprendizaje no supervisado y entrenamiento competitivo, lo cual quiere decir que no se necesita intervención humana durante el mismo y que se necesita saber muy poco sobre las características de la información de entrada; ${ }^{26}$ en el aprendizaje competitivo las neuronas compiten unas con otras con el fin de llevar a cabo una tarea dada. Se pretende que cuando se presente a la red un patrón de entrada sólo una de las neuronas de salida (o un grupo de vecinas) se active; este aprendizaje categoriza los datos que se introducen en la red. Se clasifican los valores similares en la misma categoría y por tanto deben activar la misma neurona de salida. ${ }^{27}$

24 Kohonen, Teuvo. Data Management by Self-Organizing Maps, p. 311.

25 Los mapas auto-organizados de Kohonen (som) [en línea], p. 1. <http://halweb.uc3m.es/esp/Personal/personas/jmmarin/ esp/DM/tema5dm.pdf>

26 Sotolongo-Aguilar, Gilberto, Guzmán-SÁnCHEZ, María Victoria, CARRILLO, Humberto. VIBLIOSOM: visualización de información bibliométrica mediante el mapeo auto-organizado, p. 477-478.

27 Los mapas auto-organizados de Kohonen (som), op. cit., p. 2.

\section{Visualización de los som}

La representación de la red SOM visualiza la distancia entre neuronas adyacentes. La misma se calcula y se presenta con diferentes colores entre los nodos adyacentes; podemos decir que en los mapas cada documento o artículo ocupa un lugar en el espacio, en función de sus contenidos temáticos. Cada área del mapa refleja un contenido específico, los tópicos varían ligeramente. Las diferentes tonalidades indican la densidad de documentos, cuanto más oscuras más documentos se encuentran. ${ }^{28}$

En la figura 1 se pueden observar las neuronas indicadas por un punto negro. La representación revela que existe una clase separada en la esquina superior derecha de la red. Las clases están separadas por una zona negra. Este resultado se logra con aprendizaje no supervisado, es decir, sin intervención humana. Enseñar a una red SOM y representarla ofrece una forma rápida de analizar la distribución de los datos.

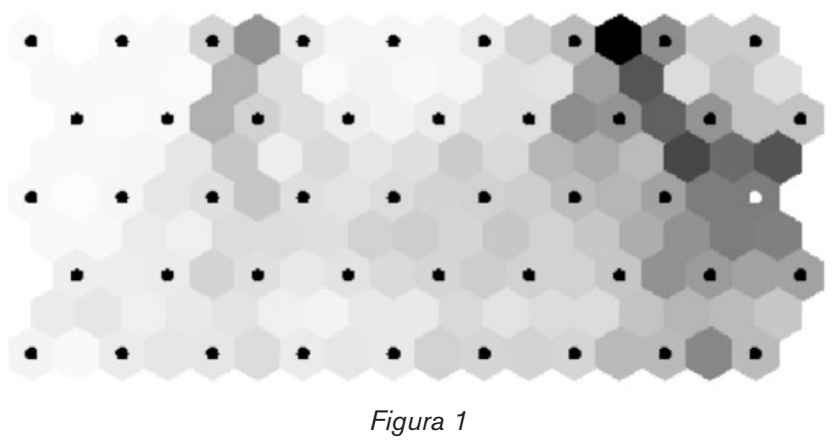

\section{Aplicaciones de los SOM}

Según Kohonen, ${ }^{29}$ a finales del año 2005 se habían registrado más de 7700 publicaciones científicas que analizan, desarrollan o aplican los SOM; la siguiente lista es un ejemplo de las principales áreas de aplicación:

\footnotetext{
28 Guzmán-SÁnchez, María Victoria, Sotolongo-Aguilar, Gilberto. Mapas tecnológicos para la estrategia empresarial: situación tecnológica de la neisseria meningitidis, p. 6.

29 Kohonen, Teuvo. Data Management by, op. cit., p. 313
} 
- Métodos estadísticos.

- El análisis exploratorio de datos estadísticos multivariados en general.

- El análisis estadístico y la organización de los textos (en los EMI y en data minning).

- Análisis y control industrial.

- Telecomunicaciones.

- Análisis financieros en general.

- Análisis y aplicaciones biomédicas en general.

El principal atractivo de los SOM se encuentra en la aplicabilidad a grandes conjuntos de datos, incluso si los datos son demasiado grandes para ser cargados de una sola vez se puede entrenar en el mapa secuencialmente (al azar) en subconjuntos de datos. Una característica especialmente interesante es que estos mapas pueden mostrar el agrupamiento de los datos sin llegar explícitamente a la realización de una agrupación. ${ }^{30} \mathrm{El}$ mapa resultante consiste por lo general en una red o rejilla de dos dimensiones, sobre la que se proyecta una distribución de elementos de entrada no lineal. El mapeo tiende a preservar las relaciones topológica-métricas entre elementos de entrada. ${ }^{31}$

\section{Los mapas auto organizados aplicados a los EMI}

Los EMI constituyen un conjunto de disciplinas que estudian los aspectos cuantitativos de la información registrada. Para ello se ha creado una serie de modelos estadísticos que aportan datos numéricos sobre el comportamiento de la actividad científica. También se han adaptado modelos de otras disciplinas para facilitar los análisis y representar los resultados desarrollados a partir de la Bibliometría. Los mapas autoorganizados (SOM) son una de estas herramientas.

En los estudios métricos la aplicación de las redes neuronales, y específicamente los SOM, está asociada en lo fundamental con la clasificación de información, o sea, la for-

30 Wehrens, Ron. Self-Organizing Maps, p. 73-74.

31 KoHonen, Teuvo. Fast Evolutionary Learning with Batch-Type Self-Organizing Maps, p. 153. mación de conglomerados y su representación en mapas bidimensionales de conceptos, y más específicamente con el descubrimiento de información. Este último vinculado con la recuperación de la información con "ruido" e incompleta o con el tratamiento de información que incluye diferentes tipos de datos (números, texto, registros estructurados, etc.)

Las investigaciones bibliométricas, a través de la utilización de las redes neuronales, incursionan en:

- selección de variables,

- clasificación de información o formación de cluster,

- regresión,

- relaciones entre variables,

- cambios y desviaciones,

- representación de las variables. ${ }^{32}$

\section{Ejemplo de mapas creados con los softwares Viscovery SOMine ${ }^{\circledR}$; vos Viewer y Pajek}

Los cuatro mapas (mapas 1, 2, 3 y 4) adelante presentados, fueron desarrollados con Viscovery somine; estos mapas reflejan la producción científica de México sobre Nanociencia y Nanotecnología ( $N \& N$ ) de los años 2005, 2007, 2009, 2011; la información para la elaboración de los mapas fue tomada de la WoS (Web of Science), en ellos se representa de manera gráfica cómo ha desplazado la investigación sobre $\mathrm{N} \& \mathrm{~N}$, además de presentarnos cómo en los diferentes años se ha incrementado; por ejemplo, en 2005 sobre el tema que más se investigó fue sobre nanopartículas con 19 artículos, para 2007 hubo un incremento del 50\% de artículos publicados sobre esta temática (30) y para 2009 el incremento es aún mayor ya que se publicaron 70 artículos, 250\% más artículos que en el año 2005; finalmente en 2011 el incremento en relación al primer año es relevante de mencionar ya que crece un $225 \%$, aunque hay un decremento de 5 artículos en relación con lo publicado en el año 2009.

32 Sotolongo-Aguilar, Gilberto, Guzmán-Sánchez, María Victoria. Aplicaciones de las redes neuronales: el caso de la bibliometría, p. 31. 


\section{MAPA TEMA / AÑO 2005}

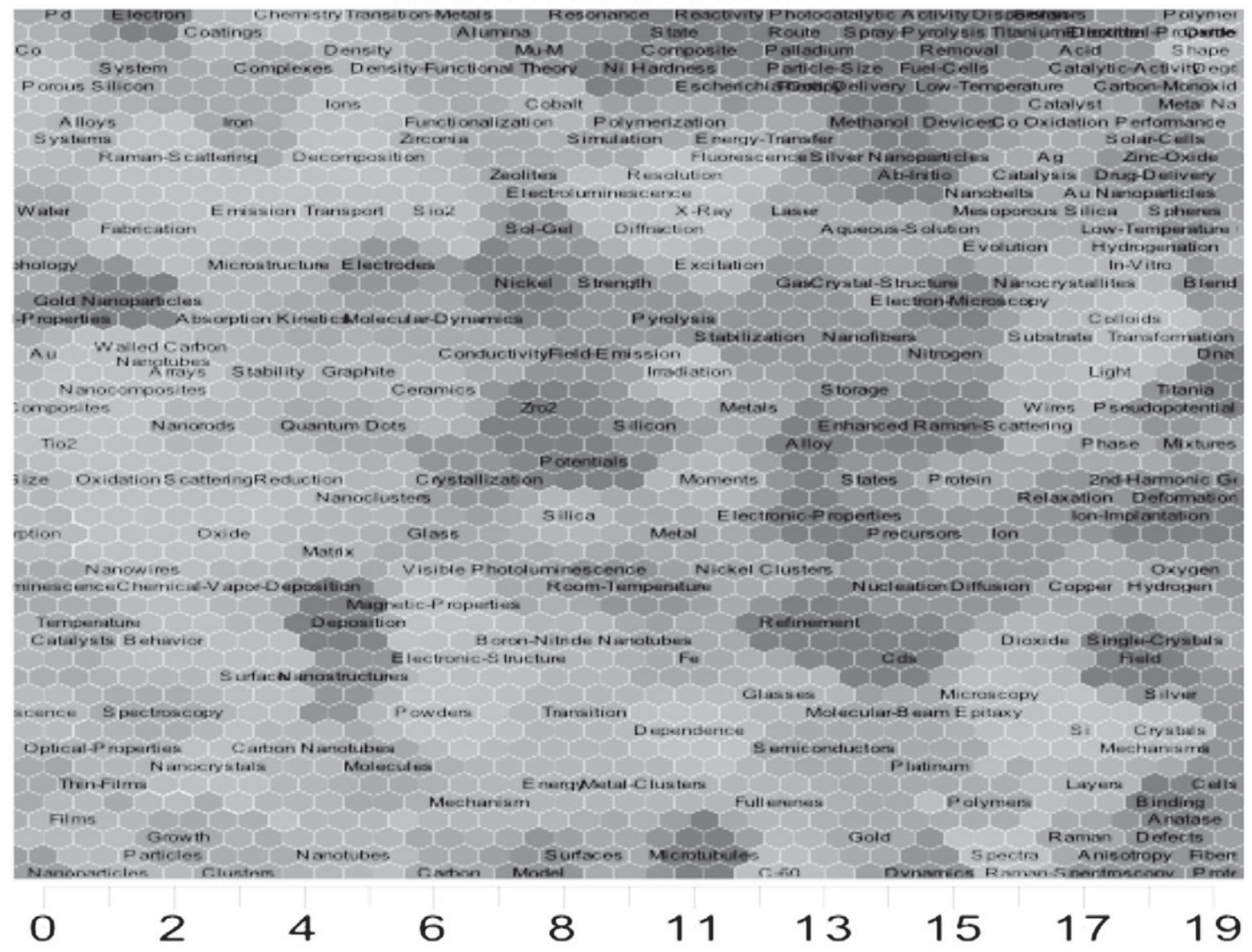

Mapa 1. Fuente: elaborado por los autores.

\section{MAPA TEMA / AÑO 2007}

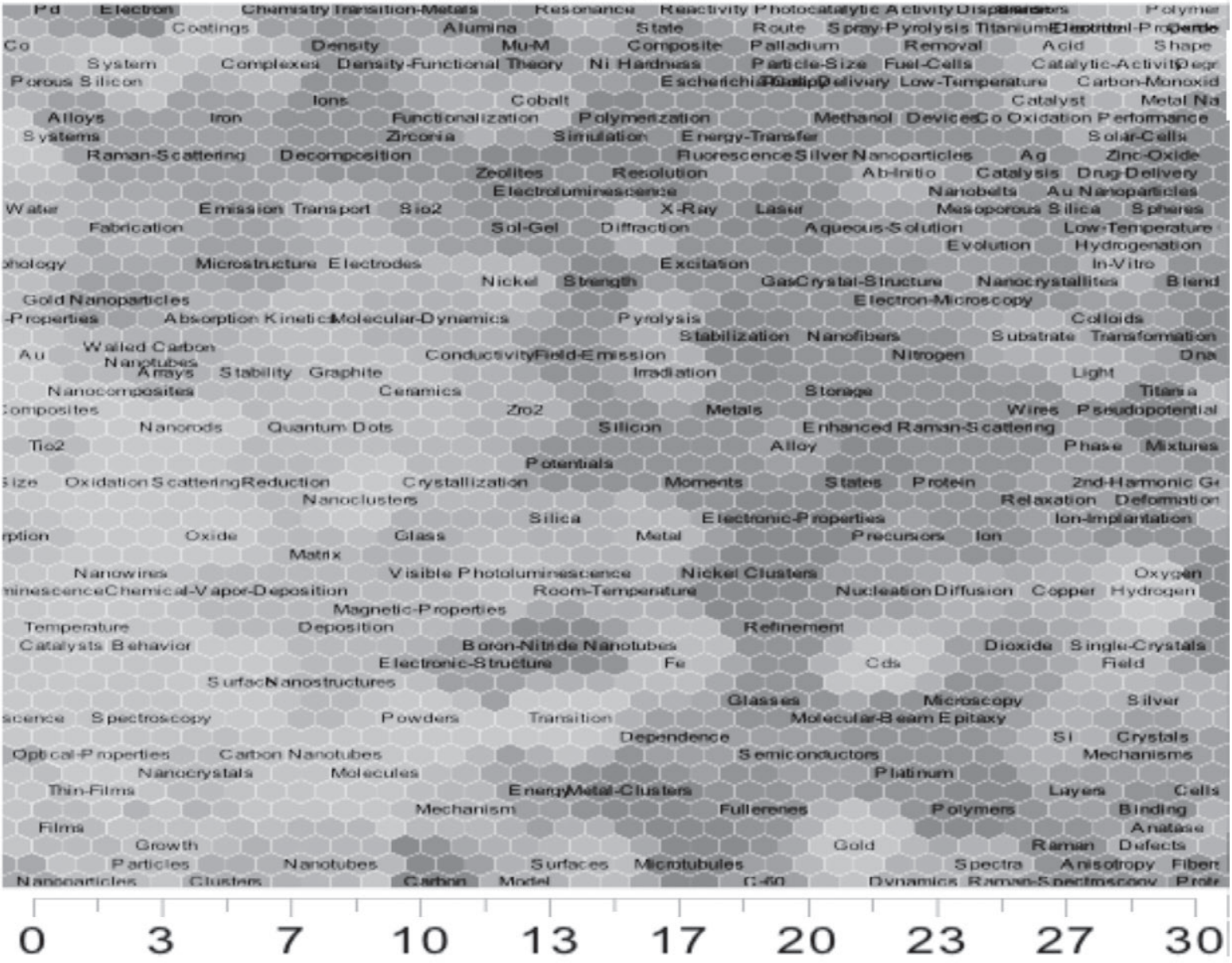

Mapa 2. Fuente: elaborado por los autores. 
MAPA TEMA / AÑO 2009

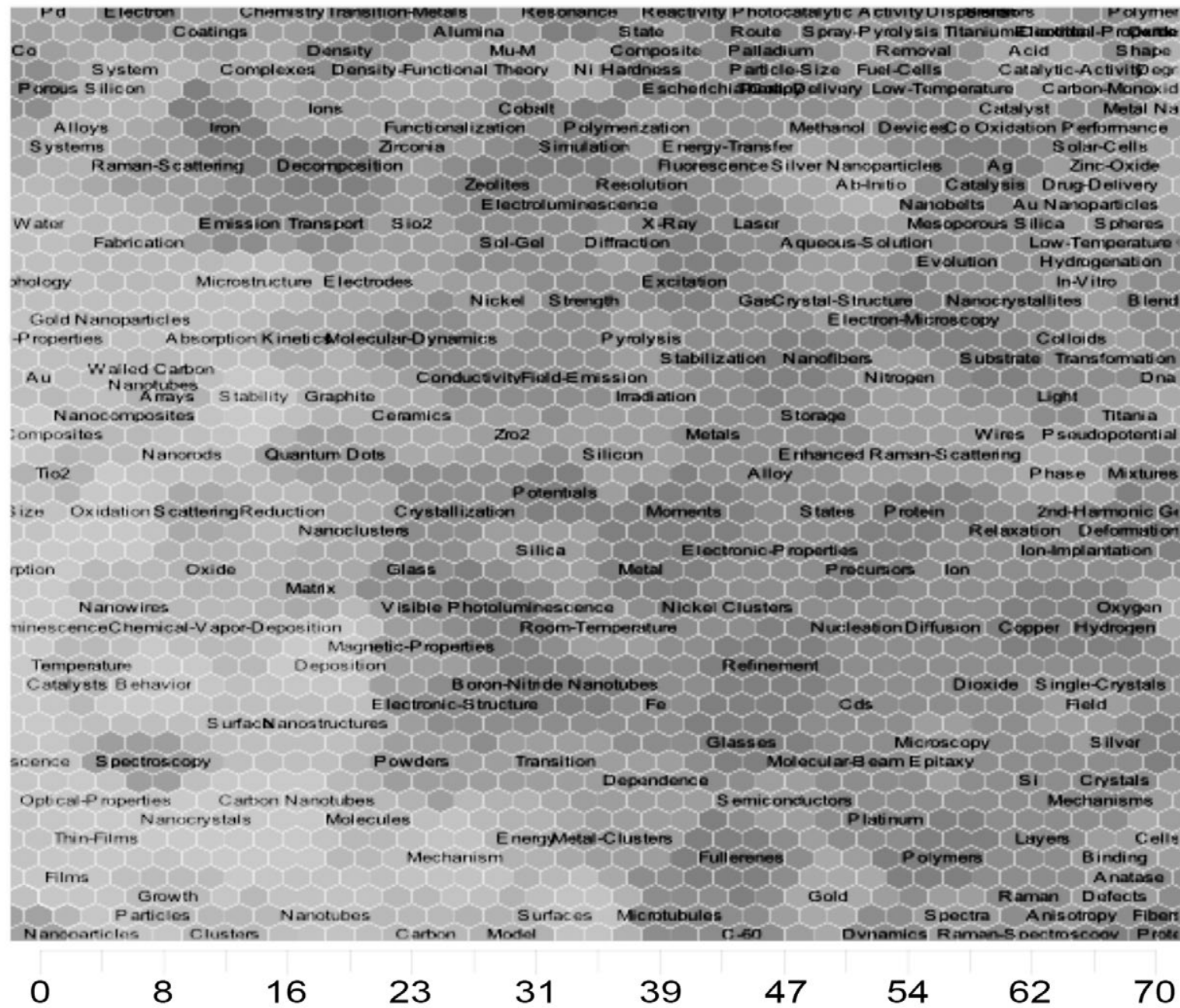

Mapa 3. Fuente: elaborado por los autores.

\section{MAPA TEMA / AÑO 2011}

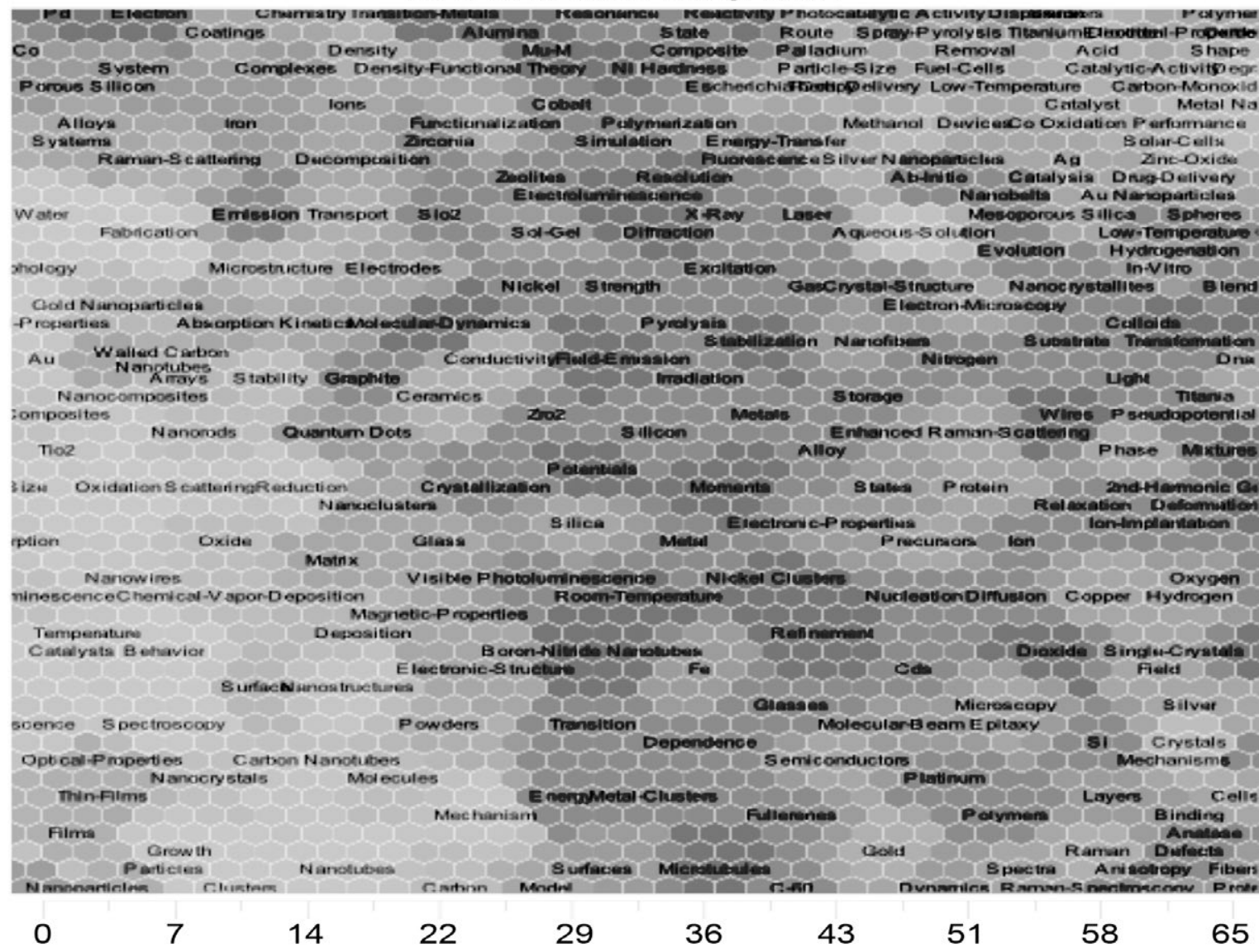


El mapa 5, que se muestra a continuación, proporciona una visión general de la estructura del mundo científico. Muestra las relaciones de las 5000 revistas científicas más importantes. El mapa se construyó utilizando Vosviewer y se basa en los datos de co-citación. Las citas fueron obtenidas de la Web of Science en el año de 2007 y refieren a los artículos publicados entre los años 1997 y 2006.

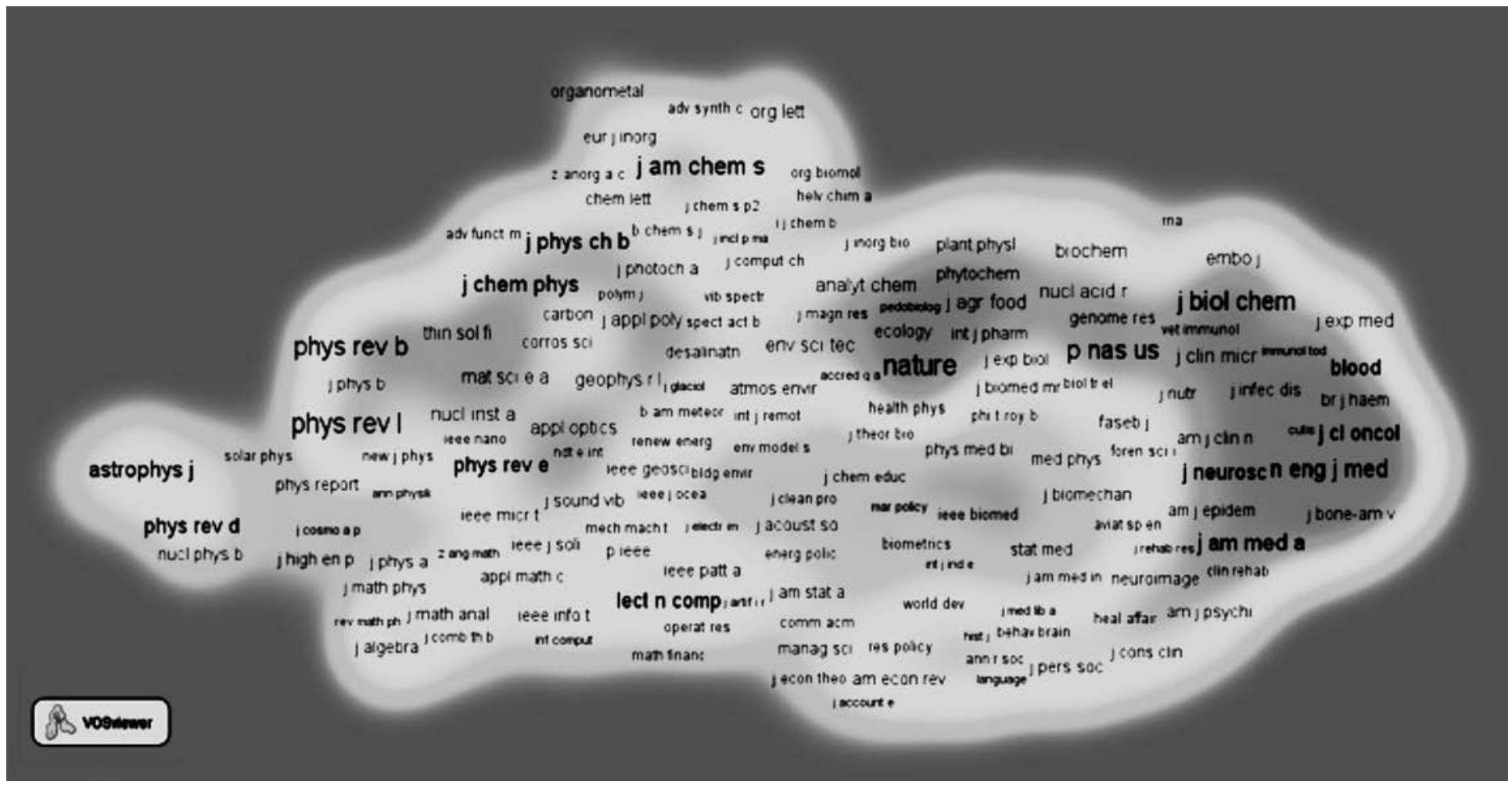

Mapa 5. Sobre las revistas científicas más importantes. Tomado de: http://www.vosviewer.com/

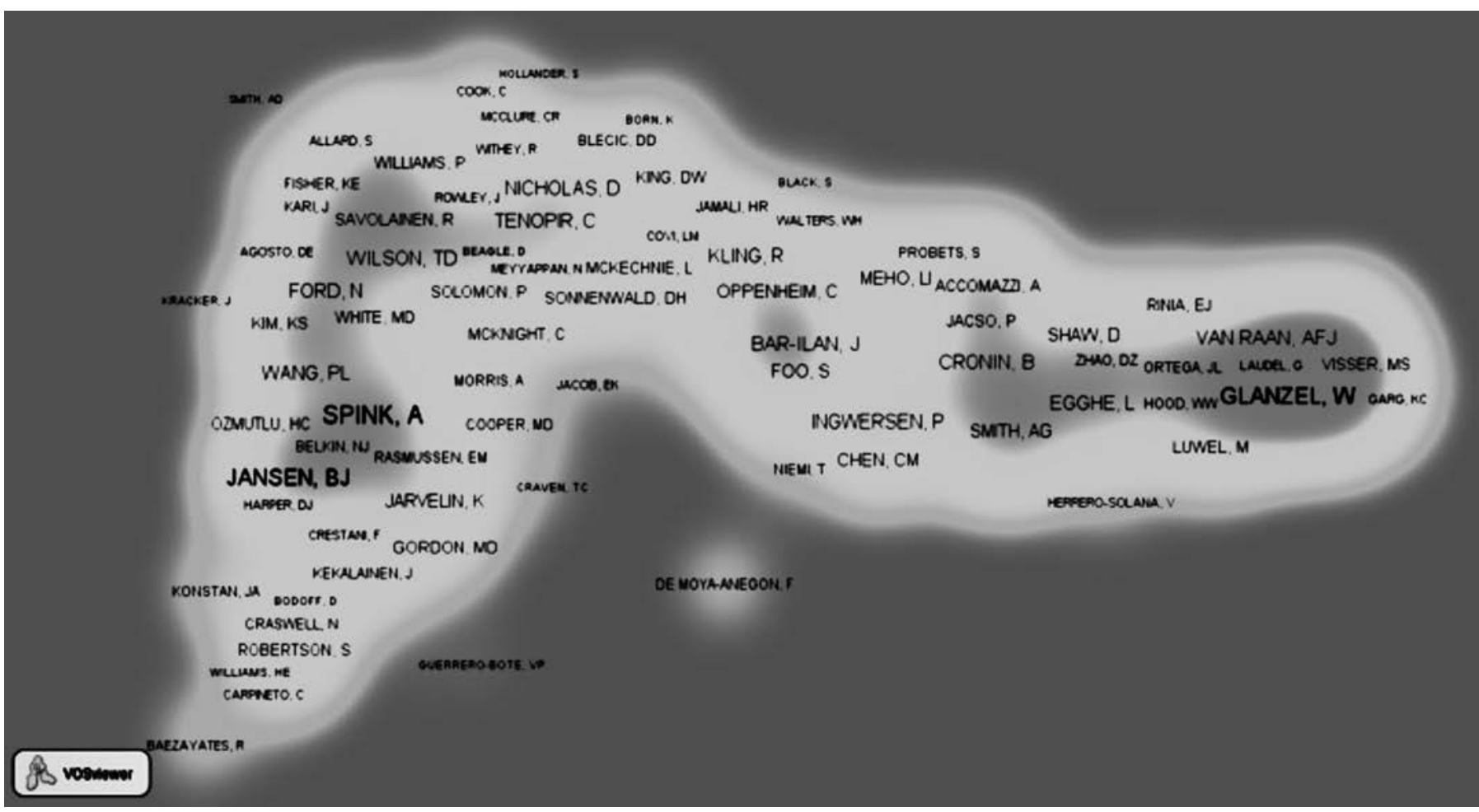

Mapa 6. Sobre los autores más citados en el campo de ciencias de la información. Tomado de: http://www.vosviewer.com/ 
La siguiente figura (mapa 6) muestra el mapeo combinado y la agrupación de las 1242 publicaciones más citadas que aparecieron en el campo de las ciencias de la información en el período 1999-2008. Las publicaciones están etiquetadas con el nombre del primer autor.

El mapa 7, desarrollado con Pajek, representa la colaboración de México en la publicación de artículos científicos relacionados con N\&N y abarcan el período 1990-2008; la base de datos utilizada para el desarrollo del presente mapa fue WoS. Como podemos observar, los autores mexicanos publican en primera instancia con científicos de Estados Unidos; un segundo bloque de colaboración lo representan países de la Comunidad Económica Europea, como son España, Francia, Italia, Inglaterra, Alemania, Irlanda.

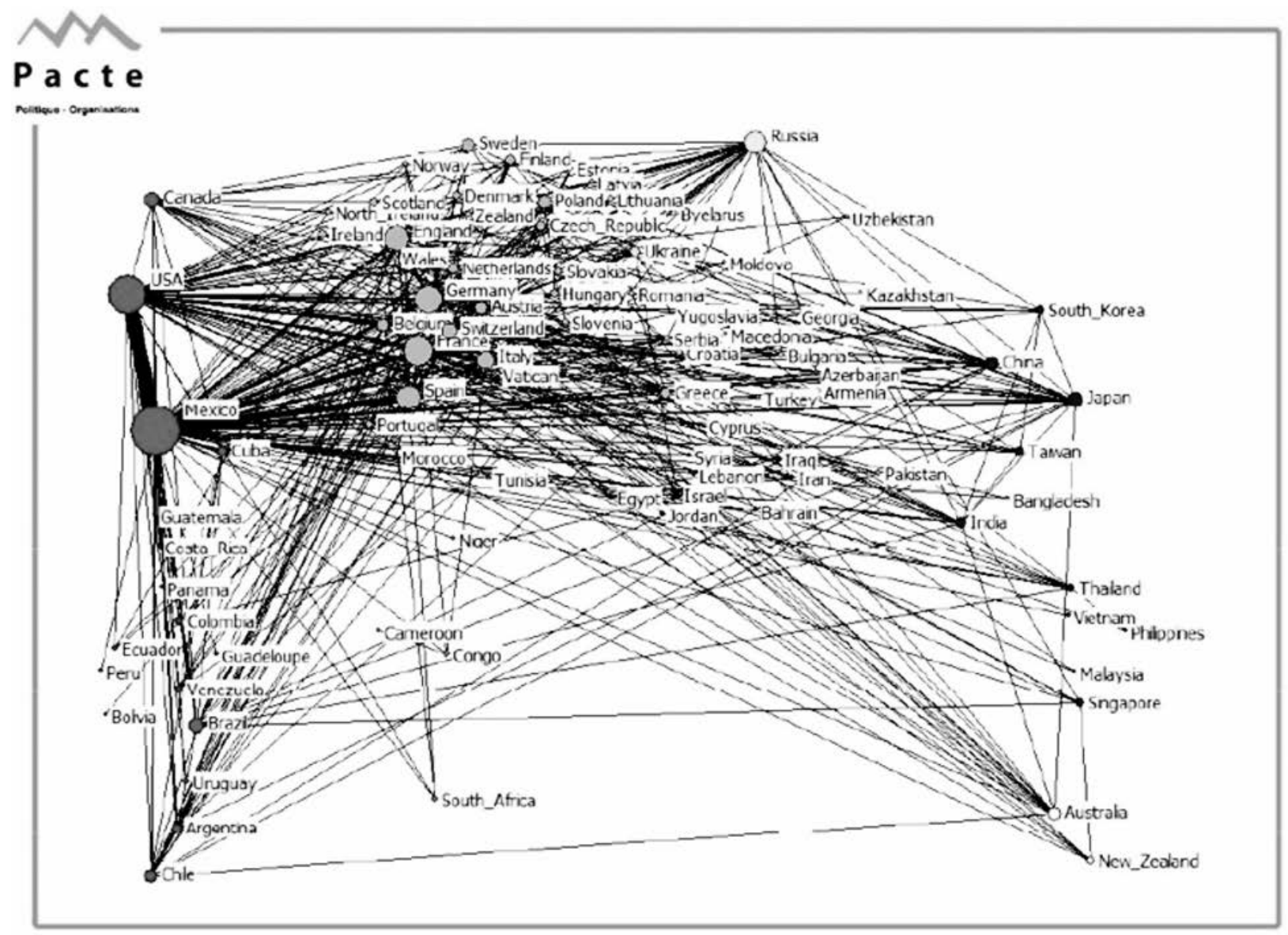

Mapa 7. Tomado de: http://www.transdisciplinario.cinvestav.mx/Portals/O/SiteDoc/Slides11a12/RoblesBelmont.pdf

\section{Conclusiones}

Como se puede observar, los mapas métricos de información son una herramienta heurística útil que proporciona una estructura visible y organizada de grandes flujos de información.

Una de las ventajas de utilizar un mapa de la ciencia es que permite navegar de un tema a otro a través de una cadena múltiple de documentos claves y afines a un tema, y permite crear así una visualización de un conjunto de datos asociativos. 
La delimitación de perfiles de actividades de investigación a partir de la categorización temática, definida a través de conjuntos de documentos, es una aproximación que permite identificar y caracterizar con suficiente detalle los subcampos de un determinado dominio o tema en una dimensión media. El enfoque elegido proporciona unos mapas que representan de forma útil la estructura reciente de la literatura científica.

Finalmente, podemos decir que el análisis de información con los mapas de la ciencia, apoyados en los estudios métricos de información, permite representar gráficamente las relaciones entre documentos que las disciplinas o campos científicos concretos publican. Éstos muestran las subáreas de investigación en las que la disciplina ha estado centrada a lo largo de los años para de este modo identificar, analizar y visualizar la estructura intelectual, así como la evolución temporal en la que se están desarrollando las disciplinas analizadas. co

\section{Obras Consultadas}

Alonso Moreno, Álvaro, González Hernández, Octavio Miguel, Lavera Ulloa, Iván. Proyecto Krowface: interfaz de simulación de redes sociales [en línea]. Sistemas Informáticos, Curso 2011-2012. <http://eprints. ucm.es/16105/1/MEMORIA_FINAL_ENTREGA. pdf $>$ [Consulta: enero 2013].

Burillo López, Fernando, Jiménez Ramiro, Alberto, Rico Teixeira, Ignacio. Proyecto Krowdix 2.0 [en línea]: sistema de simulación y análisis de redes sociales. Sistemas Informáticos, Curso 2009-2010. Universidad Complutense de Madrid, Facultad de Informática. <http://eprints.ucm.es/11276/1/ memoria_SI_Final.pdf> [Consulta: enero 2013].

Buter, Reindert K., Noyons, Ed C. M., VAN Mackelenbergh, M., Laine, T. Combining concept maps and bibliometric maps: First explorations. Scientometrics, 2006, vol. 66, no. 2, p. 377-87.

Chen, Kaihua, Guan, Jiancheng. A bibliometric investigation of research performance in emerging nanobiopharmaceuticals. Journal of Informetrics, April 2011, vol. 5, no. 2, p. 233-47.

Chen, Nian-Shing, Kinshuk, Wei, Chung-Wang, Chen, Hong-Jhe. Mining e-Learning domain concept map from academic articles. Computers o Education, Apr. 2008, vol. 50, no. 3, p. 1009-1021.
Grauwin, Sebastián, Jensen, Pablo. Mapping scientific institutions. Scientometrics, 2011, vol. 89, no. 3, p. 943-54.

GuZMÁn-SÁnchez, María Victoria, SotolongoAguilar, Gilberto. Mapas tecnológicos para la estrategia empresarial: situación tecnológica de la neisseria meningitidis. $A C I M E D, 2002$, vol. 10, no. 4, p. 1-19.

Janecek, Paul, Pu, Pearl. An evaluation of semantic fisheye views for opportunistic search in an annotated image collection. International Journal on Digital Libraries, 2005, vol. 5, no. 1, p. 42-56.

KLavans, Richard, Boyack, Kevin W. Toward a consensus map of science. Journal of the American Society for Information Science and Technology, 2009, vol. 60, no. 3, p. 455-76.

Kohonen, Teuvo. Data Management by Self-Organizing Maps. En: Computational Intelligence: Research Frontiers IEEE World Congress on Computational Intelligence, wcci 2008, Hong Kong, China, June 1-6, 2008, Plenary/Invited Lectures. Jacek M. Zurada, Gary G. Yen, Jun Wang, editors. Berlin: Springer, 2008. Lecture Notes in Computer Science, 2008, Volume 5050, p. 309-332.

Fast Evolutionary Learning with Batch-Type Self-Organizing Maps. Neural Processing Letters, 1999, vol. 9, no. 2, p. 153-62. 
Luiz Pinto, Adilson, Moreiro GonZÁlez, José Antonio, Oliveira de Mera Gusmão, Alexandre. Análisis de redes sociales a partir de recursos web y de bases de datos especializadas en literatura científica. Anales de Documentación, 2009, vol. 12, p. 139-58.

Macías-Chapula, César A. Papel de la informetría y de la cienciometría y su perspectiva nacional e internacional. ACIMED, 2001, vol. 9, supl. 4, p. 35-41.

Los mapas auto-organizados de Kohonen (SOM) [en línea]. $<$ http://halweb.uc3m.es/esp/Personal/personas/ jmmarin/esp/DM/tema5dm.pdf> [Consulta: marzo 2012].

Miguel, Sandra Edith, Caprile, Lorena, JorqueraVIDAL, Israel. Análisis de co-términos y de redes sociales para la generación de mapas temáticos. El profesional de la información, 2008, vol. 17, no. 6, p. 637-646.

Noyons, Ed C. M. Bibliometric mapping of science in a science policy context. Scientometrics, 2001, vol. 50, no. 1 , p. 83-98.

Real Academia Española. Diccionario de la lengua española [en línea]. 22 ed. <http://lema.rae.es/ drae/> [Consulta: julio 2012].

Sambrano, Jazmin, Steiner, Alicia. Mapas mentales: agenda para el éxito. México: Alfaomega, 2004. 170 p.

SmaLL, Henry. Visualizing science by citation mapping. Journal of the American Society for Information Science, 1999, vol. 50, no. 9, p. 799-813.

Solutecia [en línea]. Soluciones Tecnológicas en Inteligencia Artificial. <http://portal.solutecia.com/ producto-sofia/129-mapa-de-conocimiento $>$ [Consulta: 2011].

Sotolongo-Aguilar, Gilberto, GuZmÁn-SÁnCheZ, María Victoria. Aplicaciones de las redes neuronales: el caso de la bibliometría. Ciencias de la Información, 2001, vol. 32, no. 1, p. 27-34.
Sotolongo-Aguilar, Gilberto, GuZmán-SánCheZ, María Victoria, CARRILlo, Humberto. viBLIOSOM: visualización de información bibliométrica mediante el mapeo auto-organizado. Revista Española de Documentación Cientifica, 2002, vol. 25, no. 4, p. 477-484.

TAgue-Sutcliffe, Jean. Introducción a la informetría. ACIMED, septiembre-diciembre 1994, vol. 3, no. 2, p. 26-35.

Tergan, Sigmar-Olaf. Digital Concept Maps for Managing Knowledge and Information. En: Knowledge and Information Visualization: Searching for Synergies. Sigmar-Olaf Tergan, Tanja Keller, editors. Berlin: Springer, 2005, p. 185-204.

Van Eck, Nees Jan, Waltman, Ludo, Noyons, Ed C. M., Buter, Reindert K. Automatic term identification for bibliometric mapping. Scientometrics, 2010, vol. 82 , no. 3, p. 581-596.

Van Eck, Nees Jan, Waltman, Ludo. vosviewer Manual [en línea]. Universiteit Leiden: Erasmus Universiteit Rotterdam, 1 January 2013. <http:// www.vosviewer.com/documentation/Manual_ VOSviewer_1.5.4.pdf> [Consulta: febrero 13, 2013].

Viscovery Somine 5.2 Explorative data mining based on SOMs and statistics [en línea] Viena, 2012. <http://www. viscovery.net/welcome> [Consulta: Febrero 2012]

Vosviewer [en línea]. Universiteit Leiden, 2013. <http:// www.vosviewer.com/> [Consulta: febrero 2013].

Wehrens, Ron. Self-Organizing Maps. En: Wehrens, Ron. Chemometrics with R: Multivariate Data Analysis in the Natural Sciences and Life Sciences. Berlin: Springer, 2011, p. 67-78.

Yoon, Byungun, LeE, Sungjoo, LeE, Gwanghee. Development and application of a keyword-based knowledge map for effective R\&D planning. Scientometrics, December 2010, vol. 85, no. 3, p. 803-820. 